Ibn Al-Haitham Jour. for Pure \& Appl. Sci. 33 (3) 2020

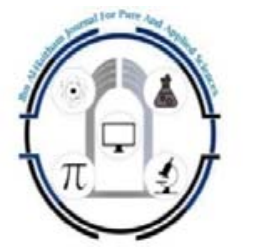

Ibn Al Haitham Journal for Pure and Applied Science Journal homepage: http://jih.uobaghdad.edu.iq/index.php/j/index

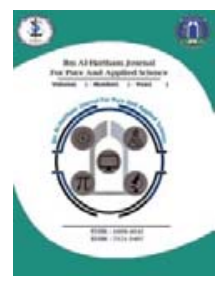

\title{
The Continuous Classical Optimal Control Problems for Triple Nonlinear Elliptic Boundary Value Problem
}

\author{
Jamil A. Al-Hawasy \\ Doaa Kateb Jasim \\ Department of Mathematics, College of Science, University of Mustansiriyah Baghdad, Iraq. \\ Jhawassy17@uomustansiriyah.edu.iq $\quad$ hawasy20@yahoo.com \\ Article history: Received 4 September 2019, Accepted 4 November 2019, Published \\ in July 2020.
}

Doi: $10.30526 / 33.3 .2477$

\begin{abstract}
In this research, our aim is to study the optimal control problem (OCP) for triple nonlinear elliptic boundary value problem (TNLEBVP). The Mint-Browder theorem is used to prove the existence and uniqueness theorem of the solution of the state vector for fixed control vector. The existence theorem for the triple continuous classical optimal control vector (TCCOCV) related to the TNLEBVP is also proved. After studying the existence of a unique solution for the triple adjoint equations (TAEqs) related to the triple of the state equations, we derive The Fréchet derivative (FD) of the cost function using Hamiltonian function. Then the theorems of necessity conditions and the sufficient condition for optimality of the constraints problem are proved.
\end{abstract}

Keywords: Triple nonlinear elliptic value problem, continuous classical optimal control vector, Mint-Browder theorem, triple adjoint equations, Fréchet derivative necessity and sufficient theorems.

\section{Introduction}

The OCP is one of the most important subject not only in mathematics, but in all branches of science, for instance, in engineering such as robotics [1]. And aeronautics [2]. In the medicine and mathematical biology, such as modeling and optimal controlling the infectious diseases [3]. In the life sciences, such as sustainable forest management [4].

In the past few decades, there were many studies and papers published in OCPs for systems that related to nonlinear ordinary differential equations [5]. or systems related to nonlinear partial differential equation (NLPDEqs) either of: a hyperbolic type [6]. Or of a parabolic type [7]. Or of an elliptic type [8]. 
or OCP are related to couple of NLPDEqs of: a hyperbolic [9]. Or of hyperbolic but include a boundary control [10]. Or of a parabolic type [11].Or of a parabolic type but includes a boundary control [12]. Or of an elliptic type [13]. Of an elliptic type that includes a Numann boundary control [14]. While other papers deals with the optimal control problems that are related to triple linear partial differential equation of : an elliptic type [15]. Or of an parabolic type [16].

In this work, the Minty-Browder theorem is used to prove the existence theorem for a unique solution (continuous state vector) for the TNLEBVP for fixed TCCOCV, and to state and prove the theorem for the existence TCCOCV related to the TNLEBVP, so as the theorem of the existence of a unique solution of the TAEqs related to the TNLEBVP. The FD of the cost function is derived. At the end the theorem of necessity conditions is stated and proved so as is the sufficient condition theorem for optimality of the constrained problem.

\section{The Problem Description}

Let $\Lambda$ be an open (bounded) connected subset in $\mathbb{R} \times \mathbb{R}$ with Lipschitz boundary $\partial \Lambda$. Consider the TCCOC of the TNLEBVP

$-B_{1} \xi_{1}+\xi_{1}-\xi_{2}-\xi_{3}+a_{1}\left(x, \xi_{1}, v_{1}\right)=a_{2}\left(x, v_{1}\right)$, in $\Lambda$

$-B_{2} \xi_{2}+\xi_{1}+\xi_{2}+\xi_{3}+p_{1}\left(x, \xi_{2}, v_{2}\right)=p_{2}\left(x, v_{2}\right)$, in $\Lambda$

$-B_{3} \xi_{3}+\xi_{1}-\xi_{2}+\xi_{3}+k_{1}\left(x, \xi_{3}, v_{3}\right)=k_{2}\left(x, v_{3}\right)$, in $\Lambda$

with the Dirchlet boundary condition

$\xi_{1}=\xi_{2}=\xi_{3}=0$, in $\partial \Lambda$

Where $\mathrm{B}_{\mathrm{r}} \xi_{\mathrm{r}}=\sum_{\mathrm{i}, \mathrm{j}}^{2} \frac{\partial}{\partial \mathrm{x}_{\mathrm{i}}}\left(\mathrm{b}_{\mathrm{ij}} \frac{\partial \xi_{\mathrm{r}}}{\partial \mathrm{x}_{\mathrm{j}}}\right), r=1,2,3, \mathrm{~b}_{\mathrm{ij}}=\mathrm{b}_{\mathrm{ij}}(\mathrm{x}) \in \mathrm{L}^{\infty}(\Lambda), \forall \mathrm{i}, \mathrm{j}=1,2, \mathrm{x}=\left(\mathrm{x}_{1}, \mathrm{x}_{2}\right)$ $\vec{\xi}=\left(\xi_{1}(\mathrm{x}), \xi_{2}(\mathrm{x}), \xi_{3}(\mathrm{x})\right) \in\left(\mathrm{H}_{0}^{2}(\Lambda)\right)^{3}$ is the classical solution of the system (1)-(4), $\overrightarrow{\mathrm{v}}=$ $\left(v_{1}(x), v_{2}(x), v_{3}(x)\right) \in\left(L^{2}(\Lambda)\right)^{3}$ is the CCV, the functions $a_{1}\left(x, \xi_{1}, v_{1}\right), p_{1}\left(x, \xi_{2}, v_{2}\right)$ and $\mathrm{k}_{1}\left(\mathrm{x}, \xi_{3}, \mathrm{v}_{3}\right)$ are defined on $\Lambda \times \mathbb{R} \times \mathrm{V}_{1}, \Lambda \times \mathbb{R} \times \mathrm{V}_{2}$ and $\Lambda \times \mathbb{R} \times \mathrm{V}_{3}$ respectively, and the functions $\mathrm{a}_{2}\left(\mathrm{x}, \mathrm{v}_{1}\right), \mathrm{p}_{2}\left(\mathrm{x}, \mathrm{v}_{2}\right)$ and $\mathrm{k}_{2}\left(\mathrm{x}, \mathrm{v}_{3}\right)$ are defined on $\Lambda \times \mathrm{V}_{1}, \Lambda \times \mathrm{V}_{2}$ and $\Lambda \times \mathrm{V}_{3}$ respectively with $V_{1}, V_{2}, V_{3} \subset \mathbb{R}$.

The control constraint is $\left(v_{1}, v_{2}, v_{3}\right) \in U_{1} \times U_{2} \times U_{3}=\vec{U}, \vec{U} \subset\left(L^{2}(\Lambda)\right)^{3}$, where $\vec{U}$ is the control set has the form

$$
\vec{U}=\left\{\vec{u} \in\left(L^{2}(\Lambda)\right)^{3} \mid \vec{u}=\left(u_{1}, u_{2}, u_{3}\right) \in V_{1} \times V_{2} \times V_{3}=\vec{V} \text { a.e. in } \Lambda\right\}
$$

With $\overrightarrow{\mathrm{V}} \subset \mathbb{R}^{3}$ that is convex and compact set.

\section{The cost function is}

$Y_{0}(\vec{v})=\int_{\Lambda} y_{01}\left(x, \xi_{1}, v_{1}\right) d x+\int_{\Lambda} y_{02}\left(x, \xi_{2}, v_{2}\right) d x+\int_{\Lambda} y_{03}\left(x, \xi_{3}, v_{3}\right) d x$

The state -control constraints are

$Y_{1}(\vec{v})=\int_{\Lambda} y_{11}\left(x, \xi_{1}, v_{1}\right) d x+\int_{\Lambda} y_{12}\left(x, \xi_{2}, v_{2}\right) d x+\int_{\Lambda} y_{13}\left(x, \xi_{3}, v_{3}\right) d x=0$

$\mathrm{Y}_{2}(\overrightarrow{\mathrm{v}})=\int_{\Lambda} \mathrm{y}_{21}\left(\mathrm{x}, \xi_{1}, \mathrm{v}_{1}\right) \mathrm{dx}+\int_{\Lambda} \mathrm{y}_{22}\left(\mathrm{x}, \xi_{2}, \mathrm{v}_{2}\right) \mathrm{dx}+\int_{\Lambda} \mathrm{y}_{23}\left(\mathrm{x}, \xi_{3}, \mathrm{v}_{3}\right) \mathrm{dx} \leq 0$

The set of the admissible controls is $\vec{U}_{A}=\left\{\vec{v} \in \vec{U} \mid Y_{1}(\vec{v})=0, Y_{2}(\vec{v}) \leq 0\right\}$

The TCCOC problem is to minimize the cost function (5) subject to the state constraints of

(6) and (7), i.e. to find $\vec{v}$ such that $\vec{v} \in \vec{U}_{A}$ and $Y_{0}(\vec{v})=\min _{\vec{u} \in \vec{U}_{A}} Y_{0}(\vec{u})$.

Let $\vec{W}=W_{1} \times W_{2} \times W_{3}=H_{0}^{1}(\Lambda) \times H_{0}^{1}(\Lambda) \times H_{0}^{1}(\Lambda)$, y $\|\mathrm{w}\|_{1}$ and $\|\vec{w}\|_{1}$ are denoted by the norm in $\mathrm{H}^{1}(\Lambda)$ and $\left(\left(\mathrm{H}^{1}(\Lambda)\right)^{3}\right.$ respectively, $\mathrm{y}\|w\|_{0}\left(\|\vec{w}\|_{0}\right)$ are denoted the norm in $L^{2}(\Lambda)$ 
Ibn Al-Haitham Jour. for Pure \& Appl. Sci. 33 (3) 2020

and in $\left(L^{2}(\Lambda)\right)^{3}$ respectively and the inner product in $W$ is denoted by $(w, w)$, with $\|\vec{w}\|=$ $\left\|w_{1}\right\|+\left\|w_{2}\right\|+\left\|w_{3}\right\|, \overrightarrow{\mathrm{W}^{*}}$ is dual of $\overrightarrow{\mathrm{W}}$.

\section{Weak Formulation of the TNLEBVP}

The weak form (WF) of (1)-(4) is obtained through multiplying both sides of Equations (1)(3) byw $w_{1} \in \mathrm{W}_{1}, \mathrm{w}_{2} \in \mathrm{W}_{2}$ and $\mathrm{w}_{3} \in \mathrm{W}_{3}$ respectively, then integrating the obtained equations. Finally, using the generalize Green's theorem for the 1st term in left hand side (L.H.S) of the three obtained equations, once get $\forall w_{1}, w_{2}, w_{3} \in W_{2}$

$b_{1}\left(\xi_{1}, w_{1}\right)+\left(\xi_{1}, w_{1}\right)-\left(\xi_{2}, w_{1}\right)-\left(\xi_{3}, w_{1}\right)+\left(a_{1}\left(\xi_{1}, v_{1}\right), w_{1}\right)=\left(a_{2}\left(v_{1}\right), w_{1}\right)$

$b_{2}\left(\xi_{2}, w_{2}\right)+\left(\xi_{1}, w_{2}\right)+\left(\xi_{2}, w_{2}\right)+\left(\xi_{3}, w_{2}\right)+\left(p_{1}\left(\xi_{2}, v_{2}\right), w_{2}\right)=\left(p_{2}\left(v_{2}\right), w_{2}\right)$

$b_{3}\left(\xi_{3}, w_{3}\right)+\left(\xi_{1}, w_{3}\right)-\left(\xi_{2}, w_{3}\right)+\left(\xi_{3}, w_{3}\right)+\left(k_{1}\left(\xi_{3}, v_{3}\right), w_{3}\right)=\left(k_{2}\left(v_{3}\right), w_{3}\right)$

where $\mathrm{b}_{\mathrm{r}}\left(\xi_{\mathrm{r}}, \mathrm{w}_{\mathrm{r}}\right)=\int_{\Lambda} \sum_{\mathrm{i}, \mathrm{j}=1}^{2} \mathrm{~b}_{\mathrm{ij}} \frac{\partial \xi_{\mathrm{r}}}{\partial \mathrm{x}_{\mathrm{i}}} \cdot \frac{\partial \mathrm{w}_{\mathrm{r}}}{\partial \mathrm{x}_{\mathrm{j}}} \mathrm{dx},\left(\xi_{\mathrm{r}}, \mathrm{w}_{\mathrm{p}}\right)=\int_{\Lambda} \xi_{\mathrm{r}} \mathrm{w}_{\mathrm{p}} \mathrm{dx},\left(\Theta, \mathrm{w}_{\mathrm{r}}\right)=\int_{\Lambda} \Theta \mathrm{w}_{\mathrm{r}} \mathrm{dx}$,

with $\Theta=a_{\imath}$ or $p_{\imath}$ or $k_{\imath}, r, p=1,2,3, \imath=1,2$.

By blending to gather equations (8), (9) and (10), once get

$\mathrm{B}(\vec{\xi}, \overrightarrow{\mathrm{w}})+\left(\mathrm{a}_{1}\left(\xi_{1}, \mathrm{v}_{1}\right), \mathrm{w}_{1}\right)+\left(\mathrm{p}_{1}\left(\xi_{2}, \mathrm{v}_{2}\right), \mathrm{w}_{2}\right)+\left(\mathrm{k}_{1}\left(\xi_{3}, \mathrm{v}_{3}\right), \mathrm{w}_{3}\right)=\left(\mathrm{a}_{2}\left(\mathrm{v}_{1}\right), \mathrm{w}_{1}\right)+$

$\left(\mathrm{p}_{2}\left(\mathrm{v}_{2}\right), \mathrm{w}_{2}\right)+\left(\mathrm{k}_{2}\left(\mathrm{v}_{3}\right), \mathrm{w}_{3}\right)$

where $B(\vec{\xi}, \vec{w})=b_{1}\left(\xi_{1}, w_{1}\right)+\left(\xi_{1}, w_{1}\right)-\left(\xi_{2}, w_{1}\right)-\left(\xi_{3}, w_{1}\right)+b_{2}\left(\xi_{2}, w_{2}\right)+\left(\xi_{1}, w_{2}\right)+$

\section{Hypotheses A:}

$$
\left(\xi_{2}, w_{2}\right)+\left(\xi_{3}, w_{2}\right)+b_{3}\left(\xi_{3}, w_{3}\right)+\left(\xi_{1}, w_{3}\right)-\left(\xi_{2}, w_{3}\right)+\left(\xi_{3}, w_{3}\right)
$$

a) $B(\vec{\xi}, \vec{w})$ is coercive, i.e. $\frac{B(\vec{\xi}, \vec{\xi})}{\|\vec{\xi}\|_{1}} \geq \epsilon\|\vec{\xi}\|_{1}>0, \vec{\xi} \in \vec{W}$

b) $|\mathrm{B}(\vec{\xi}, \overrightarrow{\mathrm{w}})| \leq \epsilon_{1}\|\vec{\xi}\|_{1}\|\overrightarrow{\mathrm{w}}\|_{1}, \epsilon_{1}>0$

c) the functions $\mathrm{a}_{1}\left(\mathrm{x}, \xi_{1}, \mathrm{v}_{1}\right), \mathrm{p}_{1}\left(\mathrm{x}, \xi_{2}, \mathrm{v}_{2}\right)$ and $\mathrm{k}_{1}\left(\mathrm{x}, \xi_{3}, \mathrm{v}_{3}\right)$ are of Carathéodory type on $\Lambda \times \mathbb{R} \times \mathrm{V}_{1}, \quad \Lambda \times \mathbb{R} \times \mathrm{V}_{2}$ and $\Lambda \times \mathbb{R} \times \mathrm{V}_{3}$ respectively and satisfy the following sublinearity conditions with respect to (w.r.t.) $\left(\xi_{1}, v_{1}\right),\left(\xi_{2}, v_{2}\right)$ and $\left(\xi_{3}, v_{3}\right)$ respectively.

$$
\begin{gathered}
\left|\mathrm{a}_{1}\left(\mathrm{x}, \xi_{1}, \mathrm{v}_{1}\right)\right| \leq \vartheta_{1}(\mathrm{x})+\mathrm{c}_{1}\left|\xi_{1}\right|+\bar{c}_{1}\left|\mathrm{v}_{1}\right|,\left|\mathrm{p}_{1}\left(\mathrm{x}, \xi_{2}, \mathrm{v}_{2}\right)\right| \leq \vartheta_{2}(\mathrm{x})+\mathrm{c}_{2}\left|\xi_{2}\right|+\bar{c}_{2}\left|\mathrm{v}_{2}\right| \\
\left|\mathrm{k}_{1}\left(\mathrm{x}, \xi_{3}, \mathrm{v}_{3}\right)\right| \leq \vartheta_{3}(\mathrm{x})+\mathrm{c}_{3}\left|\xi_{3}\right|+\bar{c}_{3}\left|\mathrm{v}_{3}\right|
\end{gathered}
$$

$\forall\left(\mathrm{x}, \bar{\zeta}_{\mathrm{i}}, \mathrm{v}_{\mathrm{i}}\right) \in \Lambda \times \mathbb{R} \times \mathrm{U}_{\mathrm{i}}$ with $\vartheta_{\mathrm{i}} \in \mathrm{L}^{2}(\Lambda), \quad \mathrm{c}_{\mathrm{i}}, \overline{\mathrm{c}}_{\mathrm{i}} \geq 0, \mathrm{i}=1,2,3$.

d) $\mathrm{a}_{1}\left(\mathrm{x}, \xi_{1}, \mathrm{v}_{1}\right), \mathrm{p}_{1}\left(\mathrm{x}, \xi_{2}, \mathrm{v}_{2}\right)$ and $\mathrm{k}_{1}\left(\mathrm{x}, \xi_{3}, \mathrm{v}_{3}\right)$ are monotone w.r.t. $\xi_{1}, \xi_{2}, \xi_{3}$

respectively for each $x \in \Lambda, v_{1} \in V_{1}, v_{2} \in V_{2}, v_{3} \in V_{3}$.

e) $\mathrm{a}_{1}\left(\mathrm{x}, 0, \mathrm{v}_{1}\right)=0, \forall \mathrm{x} \in \Lambda, \mathrm{v}_{1} \in \mathrm{V}_{1}, \mathrm{p}_{1}\left(\mathrm{x}, 0, \mathrm{v}_{2}\right)=0, \forall \mathrm{x} \in \Lambda, \mathrm{v}_{2} \in \mathrm{V}_{2}$, $\mathrm{k}_{1}\left(\mathrm{x}, 0, \mathrm{v}_{3}\right)=0, \forall \mathrm{x} \in \Lambda, \mathrm{v}_{3} \in \mathrm{V}_{3}$.

f) the functions $\mathrm{a}_{2}\left(\mathrm{x}, \mathrm{v}_{1}\right), \mathrm{p}_{2}\left(\mathrm{x}, \mathrm{v}_{2}\right)$ and $\mathrm{k}_{2}\left(\mathrm{x}, \mathrm{v}_{3}\right)$ are of Carathéodory type on

$\Lambda \times V_{1}, \Lambda \times V_{2}$ and $\Lambda \times V_{3}$ respectively and satisfy the following conditions

$\left|\mathrm{a}_{2}\left(\mathrm{x}, \mathrm{v}_{1}\right)\right| \leq \vartheta_{4}(\mathrm{x})+\mathrm{c}_{4}\left|\mathrm{v}_{1}\right|, \quad\left|\mathrm{p}_{2}\left(\mathrm{x}, \mathrm{v}_{2}\right)\right| \leq \vartheta_{5}(\mathrm{x})+\mathrm{c}_{5}\left|\mathrm{v}_{2}\right|, \quad\left|\mathrm{k}_{2}\left(\mathrm{x}, \mathrm{v}_{3}\right)\right| \leq \vartheta_{6}(\mathrm{x})+\mathrm{c}_{6}\left|\mathrm{v}_{3}\right|$

$\forall\left(\mathrm{x}, \mathrm{v}_{\mathrm{i}}\right) \in \Lambda \times \mathrm{U}_{\mathrm{i}}, i=1,2,3$ with $\vartheta_{\mathrm{r}} \in \mathrm{L}^{2}(\Lambda), c_{r} \geq 0, r=4,5,6$.

Theorem 3.1 (The Minty-Browder theorem)[17]. let $\mathrm{W}$ be a reflexive Banach space and $\mathrm{D}: \mathrm{W} \rightarrow \mathrm{W}^{*}$ be a nonlinear continuous map such that

$\left(D w_{1}-D w_{2}, w_{1}-w_{2}\right)>0, \forall w_{1}, w_{2} \in W, w_{1} \neq w_{2} \quad$ and $\quad \lim _{\|w\| \rightarrow \infty} \frac{(D w, w)}{\|w\|}=\infty$

Then the equation $D \xi=$ a has a unique (solution) $\xi \in \mathrm{W}$ for every $\mathrm{a} \in \mathrm{W}^{*}$. 
Proposition 3.1 [18]. Let $a: \Lambda \times \mathbb{R}^{n} \rightarrow \mathbb{R}^{m}$ is of Carathéodory type, and the functional $A$ is defined by $A(\xi)=\int_{\Lambda} a(x, \xi(x)) d x$, where $\Lambda$ is a measurable subset of $\mathbb{R}^{n}$, and suppose that

$\|a(x, \xi)\| \leq \vartheta(x)+\eta(x)\|\xi\|^{\alpha}, \forall(x, \xi) \in \Lambda \times \mathbb{R}^{n}, \xi \in L^{P}\left(\Lambda \times \mathbb{R}^{n}\right)$

where $\vartheta \in L^{1}(\Lambda \times \mathbb{R}), \eta \in L^{\frac{P}{P-\alpha}}(\Lambda \times \mathbb{R})$, and $\alpha \in[0, P]$, if $P \in[1, \infty)$, and $\eta \equiv 0$, if $P=\infty$. Then $A$ is continuous on $L^{P}\left(\Lambda \times \mathbb{R}^{n}\right)$.

Theorem 3.2: In addition to the hypo.(A-a\&d), If at least one of the functions $a_{1}, p_{1}$ or $k_{1}$ in hypo.(A-d) is strictly monotone. Then for any fixed controlv $\in \vec{U}_{A}$, the WF (11) has a unique solution $\vec{\xi} \in \overrightarrow{\mathrm{W}}$.

Proof: let $\overline{\mathrm{D}}: \overrightarrow{\mathrm{W}} \rightarrow \overrightarrow{\mathrm{W}}^{*}$, then the WF (11) is rewriting as

$(\bar{D}(\vec{\xi}), \vec{w})=\left(\mathrm{a}_{2}\left(\mathrm{v}_{1}\right), \mathrm{w}_{1}\right)+\left(\mathrm{p}_{2}\left(\mathrm{v}_{2}\right), \mathrm{w}_{2}\right)+\left(\mathrm{k}_{2}\left(\mathrm{v}_{3}\right), \mathrm{w}_{3}\right)$

where $(\bar{D}(\vec{\xi}), \vec{w})=B(\vec{\xi}, \vec{w})+\left(a_{1}\left(\xi_{1}, v_{1}\right), w_{1}\right)+\left(p_{1}\left(\xi_{2}, v_{2}\right), w_{2}\right)+\left(k_{1}\left(\xi_{3}, v_{3}\right), w_{3}\right)$

Then $\overline{\mathrm{D}}$ satisfies the following:

i) $\overline{\mathrm{D}}$ is coercive from hypo. (A-a\&e\&d)

ii) from hypotheses (A-a\&c) and using proposition3.1 the maping $\vec{\xi} \mapsto(\bar{D}(\vec{\xi}), \vec{w})$ is continuous w.r.t. $\vec{\xi}$.

iii) from hypotheses (A-a\&b) and (i) $\bar{D}$ is strictly monotone w.r.t. $\vec{\xi}$.

Hence by Theorem3.1, there exists a unique weak solution $\vec{\xi} \in \vec{W}$ of (11).

4. Existence of the TCCOC

Lemma 4.1: If the functions $\left(\mathrm{a}_{1} \& \mathrm{a}_{2}\right),\left(\mathrm{p}_{1} \& \mathrm{p}_{2}\right)$ and $\left(\mathrm{k}_{1} \& \mathrm{k}_{2}\right)$ are Lipschitz w.r.t. $\mathrm{v}_{1}, \mathrm{v}_{2}$ and $v_{3}$ respectively, moreover the hypothesis (A). Then the transformation $\vec{v} \mapsto \vec{\xi}_{\vec{v}}$ from $\vec{U}$ to $\left(\mathrm{L}^{2}(\Omega)\right)^{3}$ is Lipschitz continuous.

Proof: let $\overrightarrow{\vec{V}}=\left(\breve{v}_{1}, \breve{v}_{2}, \breve{v}_{3}\right) \in \vec{U}$ be a given control of $\mathrm{WF}(8)-(10)$ with its corresponding state solution $\left(\check{\xi}_{1}, \check{\xi}_{2}, \check{\xi}_{3}\right)$, then by subtracting (8)-(10) from the equations which are obtained from substituting $\delta \xi_{i}=\check{\zeta}_{i}-\xi_{i}, \delta \mathrm{v}_{i}=\check{\mathrm{v}}_{i}-\mathrm{v}_{i}(i=1,2,3)$ in (8)-(10) respectively, setting $\mathrm{w}_{1}=$ $\delta \xi_{1}, \mathrm{w}_{2}=\delta \xi_{2}$ and $\mathrm{w}_{3}=\delta \xi_{3}$ and blending together the obtained equation, to give

$b_{1}\left(\delta \xi_{1}, \delta \xi_{1}\right)+\left(\delta \xi_{1}, \delta \xi_{1}\right)+b_{2}\left(\delta \xi_{2}, \delta \xi_{2}\right)+\left(\delta \xi_{1}, \delta \xi_{2}\right)+b_{3}\left(\delta \xi_{3}, \delta \xi_{3}\right)+\left(\delta \xi_{1}, \delta \xi_{3}\right)$

$+\left(a_{1}\left(\xi_{1}+\delta \xi_{1}, v_{1}+\delta v_{1}\right)-a_{1}\left(\xi_{1}, v_{1}+\delta v_{1}, \delta \xi_{1}\right)\right.$

$+\left(p_{1}\left(\xi_{2}+\delta \xi_{2}, v_{2}+\delta v_{2}\right)-p_{1}\left(\xi_{2}, v_{2}+\delta v_{2}\right), \delta \xi_{2}\right)$

$+\left(k_{1}\left(\xi_{3}+\delta \xi_{3}, v_{3}+\delta v_{3}\right)-k_{1}\left(\xi_{3}, v_{3}+\delta v_{3}\right), \delta \xi_{3}\right)$

$=-\left(a_{1}\left(\xi_{1}, v_{1}+\delta v_{1}\right)-a_{1}\left(\xi_{1}, v_{1}\right), \delta \xi_{1}\right)-\left(p_{1}\left(\xi_{2}, v_{2}+\delta v_{2}\right)-p_{1}\left(\xi_{2}, v_{2}\right), \delta \xi_{2}\right)$

$-\left(k_{1}\left(\xi_{3}, v_{3}+\delta v_{3}\right)-k_{1}\left(\xi_{3}, v_{3}\right), \delta \xi_{3}\right)+\left(a_{2}\left(v_{1}+\delta v_{1}\right), \delta \xi_{1}\right)-\left(a_{2}\left(v_{1}\right), \delta \xi_{1}\right)$

$+\left(p_{2}\left(v_{2}+\delta v_{2}\right), \delta \xi_{2}\right)-\left(p_{2}\left(v_{2}\right), \delta \xi_{2}\right)+\left(k_{2}\left(v_{3}+\delta v_{3}\right), \delta \xi_{3}\right)-\left(k_{2}\left(v_{3}\right), \delta \xi_{3}\right)$

By hypotheses (A-a\&d), one has:

$$
\begin{gathered}
\epsilon\|\overrightarrow{\delta \xi}\|_{1}^{2} \leq\left|\int_{\Lambda}\left(a_{1}\left(x, \xi_{1}, v_{1}+\delta v_{1}\right)-a_{1}\left(x, \xi_{1}, v_{1}\right)\right) \delta \xi_{1} d x\right|+\mid \int_{\Lambda}\left(p_{1}\left(x, \xi_{2}, v_{2}+\delta v_{2}\right)-\right. \\
\left.p_{1}\left(x, \xi_{2}, v_{2}\right)\right) \delta \xi_{2} d x|+| \int_{\Lambda}\left(k_{1}\left(x, \xi_{3}, v_{3}+\delta v_{3}\right)-k_{1}\left(x, \xi_{3}, v_{3}\right)\right) \delta \xi_{3} d x|+| \int_{\Lambda}\left(a _ { 2 } \left(x, v_{1}+\right.\right. \\
\left.\left.\delta v_{1}\right)-a_{2}\left(x, v_{1}\right)\right) \delta \xi_{1} d x|+| \int_{\Lambda}\left(p_{2}\left(x, v_{2}+\delta v_{2}\right)-p_{2}\left(x, v_{2}\right)\right) \delta \xi_{2} d x|+| \int_{\Lambda}\left(k _ { 2 } \left(x, v_{3}+\right.\right. \\
\left.\left.\delta v_{3}\right)-k_{2}\left(x, v_{3}\right)\right) \delta \xi_{3} d x \mid
\end{gathered}
$$

By using Lipchitz condition on $\left(a_{1} \& a_{2}\right),\left(p_{1} \& p_{2}\right)$ and $\left(k_{1} \& k_{2}\right)$ w.r.t. $v_{1}, v_{2}, v_{3}$ respectively and Cauch-Schwarz Inequality (C-S-I) of the obtained inequality, to get: 


$$
\begin{aligned}
& \|\overrightarrow{\delta \vec{\xi}}\|_{1}^{2} \leq L_{4}\left\|\delta v_{1}\right\|_{0}\left\|\delta \xi_{1}\right\|_{0}+L_{5}\left\|\delta v_{2}\right\|_{0}\left\|\delta \xi_{2}\right\|_{0}+L_{6}\left\|\delta v_{3}\right\|_{0}\left\|\delta \xi_{3}\right\|_{0} \Longrightarrow \\
& \|\overrightarrow{\delta \vec{\xi}}\|_{0} \leq \breve{L}\|\overrightarrow{\delta v}\|_{0}, \text { with } \mathrm{L}_{4}=\max \left(\frac{\mathrm{L}_{1}}{\epsilon}, \frac{\overline{\mathrm{L}}_{1}}{\epsilon}\right), L_{5}=\max \left(\frac{L_{2}}{\epsilon}, \frac{\bar{L}_{2}}{\epsilon}\right), L_{6}=\max \left(\frac{L_{3}}{\epsilon}, \frac{\bar{L}_{3}}{\epsilon}\right)
\end{aligned}
$$

\section{Hypotheses B:}

Suppose that $\mathrm{y}_{\ell i}(\forall \ell=0,1,2 \& i=1,2,3)$ is of Carathéodory type on $\Lambda \times \mathbb{R} \times \mathrm{V}_{i}$, satisfies the following condition w.r.t. $\left(\xi_{i}, \mathrm{v}_{i}\right)$, i.e.

$\left|y_{\ell i}\left(x, \xi_{i}, v_{i}\right)\right| \leq \vartheta_{\ell i}(x)+\mathfrak{c}_{\ell i} \xi_{i}^{2}+\breve{c}_{\ell i} v_{i}^{2}$, where $\left(\xi_{i}, v_{i}\right) \in \mathbb{R} \times v_{i}, \vartheta_{\ell} \in \mathrm{L}^{1}(\Lambda)$ and $\mathfrak{c}_{\ell i}, \breve{c}_{\ell i} \geq 0$.

Lemma 4.2: With hypotheses (B), the functional $\vec{v} \mapsto Y_{\ell}(\vec{v}),(\forall \ell=0,1,2$,) defines on $\left(L^{2}(\Lambda)\right)^{3}$ is continuous.

Proof: hypotheses (B) and proposition 3.1, gives that $\int_{\Lambda} y_{\ell i}\left(x, \xi_{i}, v_{i}\right) d x(\forall \ell=0,1,2, \& i=$ $1,2,3)$, is continuous on $L^{2}(\Lambda)$. Hence $Y_{\ell}(\vec{v})$ is continuous on $\left(L^{2}(\Lambda)\right)^{3}$.

Lemma 4.3 [18]. Let $y: \Lambda \times \mathbb{R}^{2} \rightarrow \mathbb{R}$ is of Carathéodory type on $\Lambda \times \mathbb{R}^{2}$, with $|y(x, \xi, v)| \leq \eta(x)+\mathbb{C} y^{2}+\mathbb{C}^{\prime} u^{2}$, where $\eta \in L^{1}(\Lambda, \mathbb{R}), \mathbb{C}, \mathbb{C}^{\prime} \geq 0$.

Then $\int_{\Lambda} y(x, \xi, v) d x$ is continuous on $L^{2}\left(\Lambda, \mathbb{R}^{2}\right)$, with $v \in V, V \subset \mathbb{R}$ is compact.

Theorem 4.1: In addition to hypotheses (A \& B), we suppose that the set of controls $\vec{U}$, with $\vec{V}$ is convex and compact, $\vec{U}_{A} \neq \phi$, where $a_{1}, p_{1}$ and $k_{1}$ are independent of $v_{1}, v_{2}$ and $v_{3}$ respectively, and $a_{2}, p_{2}$ and $k_{2}$ are linear w.r.t. $v_{1}, v_{2}$ and $v_{3}$ respectively, i.e.

$a_{1}\left(x, \xi_{1}, v_{1}\right)=a_{1}\left(x, \xi_{1}\right), p_{1}\left(x, \xi_{2}, v_{2}\right)=p_{1}\left(x, \xi_{2}\right), k_{1}\left(x, \xi_{3}, v_{3}\right)=k_{1}\left(x, \xi_{3}\right)$

$a_{2}\left(x, v_{1}\right)=a_{2}(x) v_{1} \quad, \quad p_{2}\left(x, v_{2}\right)=p_{2}(x) v_{2} \quad, \quad k_{2}\left(x, v_{3}\right)=k_{2}(x) v_{3}$, such that $\left|a_{1}\left(x, \xi_{1}\right)\right| \leq \vartheta_{1}(x)+\hat{c}_{1}\left|\xi_{1}\right|,\left|p_{1}\left(x, \xi_{2}\right)\right| \leq \vartheta_{2}(x)+\hat{c}_{2}\left|\xi_{2}\right|, \quad\left|k_{1}\left(x, \xi_{3}\right)\right| \leq \vartheta_{3}(x)+\hat{c}_{1}\left|\xi_{3}\right|$ where $\vartheta_{1}, \vartheta_{2}, \vartheta_{3} \in L^{2}(\Lambda)$ and $\hat{c}_{1}, \hat{c}_{2}, \hat{c}_{3} \geq 0,\left|a_{2}(x)\right| \leq n_{1},\left|p_{2}(x)\right| \leq n_{2}, \quad\left|k_{2}(x)\right| \leq n_{3}$ $y_{1 i}$ is independent of $v_{i}$ and $y_{\ell i}($ for $l=0,2$ and $i=1,2,3)$ is convex w.r.t. $v_{i}$ for fixed $\xi_{i}$, then there exists TCCOCV.

Proof: Since $\vec{V}$ is convex and compact, then $\vec{U}$ is weakly compact.

Since $\vec{U}_{A} \neq \varnothing$ then there exists $\vec{u} \in \vec{U}_{A}$ and a minimum sequence $\left\{\vec{v}_{n}\right\}=\left\{\left(v_{1 n}, v_{2 n}, v_{3 n}\right)\right\} \in$ $\vec{U}_{A}$, such that $\forall \vec{v}_{n} \in \vec{U}_{A}, \forall n: \lim _{n \rightarrow \infty} Y_{0}\left(\vec{v}_{n}\right)=\inf _{\vec{u} \in \vec{U}_{A}} Y_{0}(\vec{u})$.

Since $\vec{U}$ is weakly compact, then there exists a subsequence of $\left\{\vec{v}_{n}\right\}$, (let it be again $\left\{\vec{v}_{n}\right\}$ ) which converges weakly to some $\vec{v} \in \vec{U}$, i.e. $\vec{v}_{n} \rightarrow \vec{v}$ weakly in $\left(L^{2}(\Lambda)\right)^{3}$ and $\left\|\vec{v}_{n}\right\|_{0} \leq \tilde{c}, \forall n$.

Now, by using (12), hypotheses and C-S-I, give

$$
\begin{aligned}
\epsilon\left\|\vec{\xi}_{n}\right\|_{1}^{2} & \leq(\bar{D}(\vec{\xi}), \vec{\xi})=\left(a_{2}\left(x, v_{1 n}\right), \xi_{1 n}\right)+\left(p_{2}\left(x, v_{2 n}\right), \xi_{2 n}\right)+\left(k_{2}\left(x, v_{3 n}\right), \xi_{3 n}\right) \\
& \leq\left|\left(a_{2}(x) v_{1 n}, \xi_{1 n}\right)\right|+\left|p_{2}(x) v_{2 n}, \xi_{2 n}\right|+\left|\left(k_{2}(x) v_{3 n}, \xi_{3 n}\right)\right| \\
& \leq n_{1} c_{1}\left\|\xi_{1 n}\right\|_{0}+n_{2} c_{2}\left\|\xi_{2 n}\right\|_{0}+n_{3} c_{3}\left\|\xi_{3 n}\right\|_{0} \\
& \leq\left(n_{1} c_{1}+n_{2} c_{2}+n_{3} c_{3}\right)\left\|\vec{\xi}_{n}\right\|_{1}=\omega\left\|\vec{\xi}_{n}\right\|_{1}, \text { where } \omega=\max \left(n_{1} c_{1}, n_{2} c_{2}, n_{3} c_{3}\right)>0
\end{aligned}
$$

Then $\left\|\vec{\xi}_{n}\right\|_{1} \leq \mu$, for each $n$ with $\mu=\frac{\omega}{\epsilon}>0$ ( i.e. $\vec{\xi}_{n}$ is bounded $\forall$ n)

By Alaoglu theorem(Al.Th.) [19]. there exists a subsequence of $\left\{\vec{\xi}_{n}\right\}$, (let it be again

$\left\{\vec{\xi}_{n}\right\}$ )such that $\vec{\xi}_{n} \rightarrow \vec{\xi}$ weakly in $\vec{W}$, which mean that $\vec{\xi}_{n} \rightarrow \vec{\xi}$ weakly in $\left(L^{2}(\Lambda)\right)^{3}$, then by compactness theorem(Rellich-Kondrachov [20].) $\xi_{\text {in }} \rightarrow \xi_{i}$ strongly in $\left(L^{2}(\Lambda)\right)^{3}$.Since for each $\mathrm{n}, \vec{\xi}_{n}=\left(\xi_{1 n}, \xi_{2 n}, \xi_{3 n}\right)$ satisfies $(11)$,i.e. 
$\mathrm{B}\left(\vec{\xi}_{n}, \vec{w}\right)+\left(a_{1}\left(\xi_{1 n}\right), w_{1}\right)+\left(p_{1}\left(\xi_{2 n}\right), w_{2}\right)+\left(k_{1}\left(\xi_{3 n}\right), w_{3}\right)=\left(a_{2}(x) v_{1}, w_{1}\right)+$ $\left(p_{2}(x) v_{2 n}, w_{2}\right)+\left(k_{2}(x) v_{3 n}, w_{3}\right)$

Let $\left(w_{1}, w_{2}, w_{3}\right) \in(C(\bar{\Lambda}))^{3}$, to show that (16) converges to (17), such that $B(\vec{\xi}, \vec{w})+\left(a_{1}\left(\xi_{1}, v_{1}\right), w_{1}\right)+\left(p_{1}\left(\xi_{2}, v_{2}\right), w_{2}\right)+\left(k_{1}\left(\xi_{3}, v_{3}\right), w_{3}\right)=\left(a_{2}\left(v_{1}\right), w_{1}\right)+$ $\left(p_{2}\left(v_{2}\right), w_{2}\right)+\left(k_{2}\left(v_{3}\right), w_{3}\right)$

i. Since $\xi_{\text {in }} \longrightarrow \xi_{i}$ weakly in $W_{i} \stackrel{\forall i=1,2,3}{\longrightarrow} \xi_{\text {in }} \longrightarrow \xi_{i}$ weakly in $L^{2}(\Lambda)$ and

$$
\frac{\partial \xi_{\text {in }}}{\partial x_{i}} \longrightarrow \frac{\partial \xi_{i}}{\partial x_{i}} \text { weakly in } L^{2}(\Lambda)
$$

ii. from the hypotheses on $a_{2}\left(x, \xi_{1 n}\right), p_{2}\left(x, \xi_{2 n}\right)$ and $k_{2}\left(x, \xi_{3 n}\right)$ and by using the result of lemma4.2, give that $\int_{\Omega} a_{1}\left(x, \xi_{1 n}\right) w_{1} d x, \int_{\Lambda} p_{1}\left(x, \xi_{2 n}\right) w_{2} d x$ and $\int_{\Lambda} k_{1}\left(x, \xi_{3 n}\right) w_{3} d x$ are continuous w.r.t. $\xi_{1 n}, \xi_{2 n}$ and $\xi_{3 n}$ respectively since $\xi_{\text {in }} \rightarrow \xi_{i}$ strongly in $\left(L^{2}(\Lambda)\right)^{3}$, then the L.H.S of $(16) \rightarrow$ L.H.S of (17).

Also the convergence for the R.H.S of (16) to the R.H.S of (17) is obtained through $\left(v_{i n}\right.$ $\left.\rightarrow v_{i}\right)$ weakly in $L^{2}(\Lambda),(i=1,2,3)$.

But $(C(\bar{\Lambda}))^{3}$ is dense in $\vec{W}$, which gives $\vec{\xi}_{n} \rightarrow \vec{\xi}=\vec{\xi}_{\vec{v}}$ is a solution of the state equations in $\vec{W}$.

From lemma4.2, $Y_{\ell}(\vec{V})$ is continuous on $\left(L^{2}(\Lambda)\right)^{3}$, for each $\ell=0,1,2$.

From the hypotheses on $y_{\ell i}($ for $\ell=0,1,2$ and $i=1,2,3)$, and $\xi_{\text {in }} \rightarrow \xi_{i}$ strongly in $L^{2}(\Lambda)$, then $Y_{1}(\vec{v})=\lim _{n \rightarrow \infty} Y_{1}\left(\vec{v}_{n}\right)$, hence $Y_{1}(\vec{v})=0$

Now, to prove $Y_{\ell}(\vec{v}),(\ell=0,2)$ is W.L.Sc. w.r.t. $\left(\xi_{i}, v_{i}\right),(i=1,2,3)$.

From hypotheses $\mathrm{B},\left(v_{1 n}, v_{2 n}, v_{3 n}\right) \in \vec{V}$ almost everywhere (a.e.) in $\Lambda$ and $\vec{V}$ is compact, hence $Y_{\ell}(\vec{v})$ is satisfied the hypotheses of lemma4.3, and gets that

$\int_{\Lambda} y_{\ell i}\left(x, \xi_{i n}, v_{i n}\right) d x \longrightarrow \int_{\Lambda} y_{\ell i}\left(x, \xi_{i}, v_{i n}\right) d x$

Since $y_{\ell i}\left(x, \xi_{i}, v_{i}\right)$ is convex w.r.t. $v_{i}$, then $\int_{\Lambda} y_{\ell i}\left(x, \xi_{i}, v_{i}\right) d x$ is W.L.S. w.r.t. $v_{i}$, i.e.

$\int_{\Lambda} y_{\ell i}\left(x, \xi_{i}, v_{i}\right) d x \leq \underline{\lim }_{n \rightarrow \infty} \int_{\Lambda} y_{\ell i}\left(x, \xi_{i}, v_{i n}\right) d x$

$$
\begin{aligned}
& =\varliminf_{n \rightarrow \infty} \int_{\Lambda}\left(y_{\ell i}\left(x, \xi_{i n}, v_{i n}\right)-y_{\ell i}\left(x, \xi_{i n}, v_{i n}\right)\right) d x+\varliminf_{n \rightarrow \infty} \int_{\Lambda} y_{\ell i}\left(x, \xi_{i}, v_{i n}\right) d x \\
& =\varliminf_{n \rightarrow \infty} \int_{\Lambda}\left(y_{\ell i}\left(x, \xi_{i n}, v_{i n}\right) d x\right.
\end{aligned}
$$

Hence $Y(\vec{v}) \leq \lim _{n \rightarrow \infty} Y_{0}\left(\vec{v}_{n}\right)=\lim _{n \rightarrow \infty} Y_{0}\left(\vec{v}_{n}\right)=\inf _{\vec{u} \in \vec{U}_{A}} Y_{0}(\vec{u}) \Longrightarrow \vec{v}$ is an optimal control

\section{The Necessary and the Sufficient Conditions for Optimality Hypotheses C:}

a) The functions $a_{1 \xi_{1}}, a_{1 v_{1}}, p_{1 \xi_{2}}, p_{1 v_{2}}, k_{1 \xi_{3}}, k_{1 v_{3}}$ are of the Carathéodory type on

$\Lambda \times \mathbb{R} \times \mathbb{R}$ and satisfy for $x \in \Lambda$ and $d_{i}, j_{i} \geq 0,(i=1,2,3)$ :

$$
\begin{aligned}
& \left|a_{1 \xi_{1}}\left(x, \xi_{1}, v_{1}\right)\right| \leq d_{1}, \quad\left|p_{1 \xi_{2}}\left(x, \xi_{2}, v_{2}\right)\right| \leq d_{2}, \quad\left|k_{1 \xi_{3}}\left(x, \xi_{3}, v_{3}\right)\right| \leq d_{3}, \\
& \left|a_{1 v_{1}}\left(x, \xi_{1}, v_{1}\right)\right| \leq j_{1}, \quad\left|p_{1 v_{2}}\left(x, \xi_{2}, v_{2}\right)\right| \leq j_{2}, \quad\left|k_{1 v_{3}}\left(x, \xi_{3}, v_{3}\right)\right| \leq j_{3}
\end{aligned}
$$

b) The functions $a_{2 v_{1}}, p_{2 v_{2}}, k_{2 v_{3}}$ are of the Carathéodory type on $\Lambda \times \mathbb{R}$, with $\left|a_{2 v_{1}}\left(x, v_{1}\right)\right| \leq q_{1}, \quad\left|p_{2 v_{2}}\left(x, v_{2}\right)\right| \leq q_{2}, \quad\left|k_{2 v_{3}}\left(x, v_{3}\right)\right| \leq q_{3}$

where $x \in \Lambda$ and $q_{i} \geq 0,(i=1,2,3)$.

c) The functions $y_{\ell i \xi_{i}}, y_{\ell i v_{i}}(\forall \ell=0,1,2 \& i=1,2,3)$ are of the Carathéodory 
type on $\Lambda \times \mathbb{R} \times \mathbb{R}$ and satisfy the following conditions for $\eta_{\ell i}, \hat{\eta}_{\ell i} \in L^{2}(\Lambda)$ : $\left|y_{\ell i \xi_{i}}\right| \leq \eta_{\ell i}+\Upsilon_{\ell i}\left|\xi_{i}\right|+\Upsilon_{\ell i}\left|v_{i}\right|$ And $\left|y_{\ell i v_{i}}\right| \leq \hat{\eta}_{\ell i}+\widehat{\Upsilon}_{\ell i}\left|\xi_{i}\right|+\widehat{\Upsilon}_{\ell i}\left|v_{i}\right|$, with $\Upsilon_{\ell i}, \widehat{\Upsilon}_{\ell i} \geq$ 0 ,

Theorem 5.1: With hypotheses A, B and C, the Hamiltonian is:

$$
\begin{gathered}
H(x, \vec{\xi}, \vec{\zeta}, \vec{v})=\zeta_{1}\left(a_{2}\left(x, v_{1}\right)-a_{1}\left(x, \xi_{1}, v_{1}\right)\right)+y_{01}\left(x, \xi_{1}, v_{1}\right)+\zeta_{2}\left(p_{2}\left(x, v_{2}\right)-p_{1}\left(x, \xi_{2}, v_{2}\right)\right) \\
+y_{02}\left(x, \xi_{2}, v_{2}\right)+\zeta_{3}\left(k_{2}\left(x, v_{3}\right)-k_{1}\left(x, \xi_{3}, v_{3}\right)\right)+y_{03}\left(x, \xi_{3}, v_{3}\right)
\end{gathered}
$$

The adjoint vector $\left(\zeta_{1}, \zeta_{2}, \zeta_{3}\right)=\left(\zeta_{1 v_{1}}, \zeta_{2 v_{2}}, \zeta_{3 v_{3}}\right)$ "equations "of (3.1- 3.4) are:

$-B_{1} \zeta_{1}+\zeta_{1}+\zeta_{2}+\zeta_{3}+\zeta_{1} a_{1 \xi_{1}}\left(x, \xi_{1}, v_{1}\right)=y_{01 \xi_{1}}\left(x, \xi_{1}, v_{1}\right)$, in $\Lambda$

$-B_{2} \zeta_{2}-\zeta_{1}+\zeta_{2}-\zeta_{3}+\zeta_{2} p_{1 \xi_{2}}\left(x, \xi_{2}, v_{2}\right)=y_{02 \xi_{2}}\left(x, \xi_{2}, v_{2}\right)$, in $\Lambda$

$-B_{3} \zeta_{3}-\zeta_{1}+\zeta_{2}+\zeta_{3}+\zeta_{3} k_{1 \xi_{3}}\left(x, \xi_{3}, v_{3}\right)=y_{03 \xi_{3}}\left(x, \xi_{3}, v_{3}\right)$, in $\Lambda$

$\zeta_{1}=\zeta_{2}=\zeta_{3}=0$ on $\partial \Lambda$

Then the FD of $Y_{0}$ is given by:

$\overrightarrow{\vec{Y}}_{0}(\vec{v}) \overrightarrow{\delta v}=\int_{\Lambda} H_{\vec{v}}{ }^{T} \cdot \overrightarrow{\delta v} d x, \quad H_{\vec{v}}=\left(\begin{array}{l}H_{v_{1}}(x, \vec{\xi}, \vec{\zeta}, \vec{v}) \\ H_{v_{2}}(x, \vec{\xi}, \vec{\zeta}, \vec{v}) \\ H_{v_{3}}(x, \vec{\xi}, \vec{\zeta}, \vec{v})\end{array}\right)=\left(\begin{array}{l}\zeta_{1}\left(a_{2 v_{1}}-a_{1 v_{1}}\right)+y_{1 v_{1}} \\ \zeta_{2}\left(p_{2 v_{2}}-p_{1 v_{2}}\right)+y_{2 v_{2}} \\ \zeta_{3}\left(k_{2 v_{3}}-k_{1 v_{3}}\right)+y_{3 v_{3}}\end{array}\right)$

Proof: Rewriting the TAEqs (18)-(20) by their WF and then blending them together:

$\overline{\bar{B}}(\vec{\zeta}, \vec{w})+\left(\zeta_{1} a_{1 \xi_{1}}\left(\xi_{1}, v_{1}\right), w_{1}\right)+\left(\zeta_{2} p_{1 \xi_{2}}\left(\xi_{2}, v_{2}\right), w_{2}\right)+\left(\zeta_{3} k_{1 \xi_{3}}\left(\xi_{3}, v_{3}\right), w_{3}\right)$

$\left.=\left(y_{01 \xi_{1}}\left(\xi_{1}, v_{1}\right), w_{1}\right)+\left(y_{02 \xi_{2}}\left(\xi_{2}, v_{2}\right), w_{2}\right)+y_{03 \xi_{3}}\left(\xi_{3}, v_{3}\right), w_{3}\right)$

where $\overline{\bar{B}}(\vec{\zeta}, \vec{w})=b_{1}\left(\zeta_{1}, w_{1}\right)+\left(\zeta_{1}, w_{1}\right)+\left(\zeta_{2}, w_{1}\right)+\left(\zeta_{3}, w_{1}\right)+b_{2}\left(\zeta_{2}, w_{2}\right)-\left(\zeta_{1}, w_{2}\right)+$ $\left(\zeta_{2}, w_{2}\right)-\left(\zeta_{3}, w_{2}\right)+b_{3}\left(\zeta_{3}, w_{3}\right)-\left(\zeta_{1}, w_{3}\right)+\left(\zeta_{2}, w_{3}\right)+\left(\zeta_{3}, w_{3}\right)$

The WF of the TAEqs (22) has a unique solution; this can be proved using the same way which is used to prove the WF of the state equation (11).

Now by substituting $\vec{w}=\overrightarrow{\delta \zeta}$ in (22), once has:

$$
\begin{aligned}
\bar{B}(\vec{\zeta}, \overrightarrow{\delta \zeta})+\left(\zeta_{1} a_{1 \xi_{1}}\left(\xi_{1}, v_{1}\right), \delta \zeta_{1}\right)+\left(\zeta_{2} p_{1 \xi_{2}}\left(\xi_{2}, v_{2}\right), \delta \zeta_{2}\right)+\left(\zeta_{3} k_{1 \xi_{3}}\left(\xi_{3}, v_{3}\right), \delta \zeta_{3}\right) \\
(23)=\left(y_{01 \xi_{1}}\left(\xi_{1}, v_{1}\right), \delta \zeta_{1}\right)+\left(y_{02 \xi_{2}}\left(\xi_{2}, v_{2}\right), \delta \zeta_{2}\right)+\left(y_{03 \xi_{3}}\left(\xi_{3}, v_{3}\right), \delta \zeta_{3}\right)
\end{aligned}
$$

Setting the solution $\vec{\xi}+\overrightarrow{\delta \xi}$ in (8)-(10) then subtracting (8)-(10) from those equations which are obtained by setting $(\vec{\xi}+\overrightarrow{\delta \xi})$, then setting $w_{1}=\zeta_{1}, w_{2}=\zeta_{2}, w_{3}=\zeta_{3}$ and then blending them together, to get:

$$
\begin{aligned}
& B(\overrightarrow{\delta \xi}, \vec{\zeta})+\left(a_{1}\left(\xi_{1}+\delta \xi_{1}, v_{1}+\delta v_{1}\right)-a_{1}\left(\xi_{1}, v_{1}\right), \zeta_{1}\right)+\left(p_{1}\left(\xi_{2}+\delta \xi_{2}, v_{2}+\delta v_{2}\right)-\right. \\
& \left.p_{1}\left(\xi_{2}, v_{2}\right), \zeta_{2}\right)+\left(k_{1}\left(\xi_{3}+\delta \xi_{3}, v_{3}+\delta v_{3}\right)-k_{1}\left(\xi_{3}, v_{3}\right), \zeta_{3}\right)=\left(a_{2}\left(v_{1}+\delta v_{1}\right)-a_{2}\left(v_{1}\right), \zeta_{1}\right)+ \\
& \left(p_{2}\left(v_{2}+\delta v_{2}\right)-p_{2}\left(v_{2}\right), \zeta_{2}\right)+\left(k_{2}\left(v_{3}+\delta v_{3}\right)-k_{2}\left(v_{3}\right), \zeta_{3}\right)
\end{aligned}
$$

Now, from hypo. on $a_{1}, p_{1}, k_{1}, a_{2}, p_{2}$ and $k_{2}$, using proposition 3.1 and the Mean value theorem, the FD of $a_{1}, p_{1}, k_{1}, a_{2}, p_{2}$ and $k_{2}$ are exist, once get that:

$$
\begin{aligned}
& B(\overrightarrow{\delta \xi}, \vec{\zeta})+\left(a_{1 \xi_{1}} \delta \xi_{1}+a_{1 v_{1}} \delta v_{1}, \zeta_{1}\right)+\left(p_{1 \xi_{2}} \delta \xi_{2}+p_{1 v_{2}} \delta v_{2}, \zeta_{2}\right)+\left(k_{1 \xi_{3}} \delta \xi_{3}+k_{1 v_{3}} \delta v_{3}, \zeta_{3}\right) \\
& =\left(a_{2 v_{1}} \delta v_{1}, \zeta_{1}\right)+\left(p_{2 v_{2}} \delta v_{2}, \zeta_{2}\right)+\left(k_{2 v_{3}} \delta v_{3}, \zeta_{3}\right)+\tilde{\varepsilon}(\overrightarrow{\delta \Xi})\|\overrightarrow{\delta \Xi}\|_{0}
\end{aligned}
$$

where $\tilde{\varepsilon}(\overrightarrow{\delta \Xi})\|\overrightarrow{\delta \Xi}\|_{0}=\tilde{\varepsilon}(\overrightarrow{\delta \xi}, \overrightarrow{\delta v})\|\overrightarrow{\delta \vec{\xi}}\|$,

From the Minkowiski inequality and lemma 4.1, once obtain that: 
$\tilde{\varepsilon}(\overrightarrow{\delta \Xi})=\tilde{\varepsilon}(\overrightarrow{\delta \xi}, \overrightarrow{\delta v})=\bar{\varepsilon}_{1}(\overrightarrow{\delta v}), \quad\|\overrightarrow{\delta \vec{\Xi}}\|_{0}=\left\|\begin{array}{l}\delta \xi_{1} \\ \delta v_{1}\end{array}\right\| \leq c\|\overrightarrow{\delta v}\| \Longrightarrow$
$\tilde{\varepsilon}(\overrightarrow{\delta \Xi})\|\overrightarrow{\delta \Xi}\|_{0}=\tilde{\varepsilon}_{1}(\overrightarrow{\delta v})\|\overrightarrow{\delta v}\|_{0}, \quad$ where $\tilde{\varepsilon}_{1}(\overrightarrow{\delta v}) \longrightarrow 0$, and $\|\overrightarrow{\delta v}\|_{0} \rightarrow 0$ as $\overrightarrow{\delta v} \longrightarrow 0$

Hence

$$
\begin{aligned}
& B(\overrightarrow{\delta \xi}, \vec{\zeta})+\left(a_{1 \xi_{1}} \delta \xi_{1}+a_{1 v_{1}} \delta v_{1}, \zeta_{1}\right)+\left(p_{1 \xi_{2}} \delta \xi_{2}+p_{1 v_{2}} \delta v_{2}, \zeta_{2}\right)+\left(k_{1 \xi_{3}} \delta \xi_{3}+k_{1 v_{3}} \delta v_{3}, \zeta_{3}\right)= \\
& \left(a_{2 v_{1}} \delta v_{1}, \zeta_{1}\right)+\left(p_{2 v_{2}} \delta v_{2}, \zeta_{2}\right)+\left(k_{2 v_{3}} \delta v_{3}, \zeta_{3}\right)+\tilde{\varepsilon}_{1}(\overrightarrow{\delta v})\|\overrightarrow{\delta v}\|_{0}
\end{aligned}
$$

Now, from definition of the FD, hypotheses on $y_{\ell i}(\ell=0,2, i=1,2,3)$ and by using the result of lemma 4.1, once obtain that:

$Y_{0}(\vec{v}+\overrightarrow{\delta v})-Y_{0}(\vec{v})=\int_{\Lambda}\left(y_{01 \xi_{1}}\left(\xi_{1}, v_{1}\right) \delta \xi_{1}+y_{01 v_{1}}\left(\xi_{1}, v_{1}\right) \delta v_{1}\right) d x+\int_{\Lambda}\left(y_{02 \xi_{2}}\left(\xi_{2}, v_{2}\right) \delta \xi_{2}+\right.$

$\left.y_{02 v_{2}}\left(\xi_{2}, v_{2}\right) \delta v_{2}\right) d x+\int_{\Lambda}\left(y_{03 \xi_{3}}\left(\xi_{3}, v_{3}\right) \delta \xi_{3}+y_{03 v_{3}}\left(\xi_{3}, v_{3}\right) \delta v_{3}\right) d x+\tilde{\varepsilon}(\overrightarrow{\delta v})\|\overrightarrow{\delta v}\|_{0}$

where $\tilde{\varepsilon}(\overrightarrow{\delta v}) \rightarrow 0$, and $\|\overrightarrow{\delta v}\|_{0} \rightarrow 0$ as $\overrightarrow{\delta v} \rightarrow 0$

By subtracting (23) from (25b), and substituting the rustle in (26), once get

$Y_{0}(\vec{v}+\overrightarrow{\delta v})-Y_{0}(\vec{v})=\int_{\Lambda}\left(\zeta_{1}\left(a_{2 v_{1}}-a_{1 v_{1}}\right)+y_{01 v_{2}}\right) \delta v_{1} d x+\int_{\Lambda}\left(\zeta_{2}\left(p_{2 v_{2}}-p_{1 v_{2}}\right)+\right.$

$\left.y_{02 v_{2}}\right) \delta v_{2}+\int_{\Lambda}\left(\zeta_{3}\left(k_{2 v_{3}}-k_{1 v_{3}}\right)+y_{03 v_{3}}\right) \delta v_{3} d x+\tilde{\varepsilon}(\overrightarrow{\delta v})\|\overrightarrow{\delta v}\|_{0}$

Then from FD, we have that $\overrightarrow{\vec{Y}}_{0}(\vec{v}) \overrightarrow{\delta v}=\int_{\Lambda} H_{\vec{v}}{ }^{T} \cdot \overrightarrow{\delta v} d x$.

Note: In the proof of the theorem 5.1, we find the FD for the functional $Y_{0}$, so the same technique is used to find the FD for $Y_{1}$ and $Y_{2}$.

\section{Theorem 5.2: Optimality Necessary Conditions}

(a) With hypotheses $\mathrm{A}, \mathrm{B}$ and $\mathrm{C}$, assume $\vec{U}$ is convex, if $\vec{v} \in \vec{U}_{A}$ is optimal, then there exist multipliers $\lambda_{\ell} \in \mathbb{R},\left(\ell=0,1,2\right.$ with $\left.\lambda_{0}, \lambda_{2} \geq 0, \sum_{\ell=0}^{2}\left|\lambda_{\ell}\right|=1\right)$, such that the following The Kuhn- Tucker- Lagrange's Multipliers (K.T.L) are satisfied:

$\int_{\Lambda} H_{\vec{v}}{ }^{T} \cdot \overrightarrow{\delta v} d x \geq 0, \forall \vec{u} \in \vec{U}, \overrightarrow{\delta v}=\vec{u}-\vec{v}$

where $y_{i}=\sum_{\ell=0}^{2} \lambda_{\ell} y_{\ell i}$ and $\zeta_{i}=\sum_{\ell=0}^{2} \lambda_{\ell} \zeta_{\ell i},(i=1,2,3)$ in the definition of $H$, and also

$\lambda_{2} Y_{2}(\vec{v})=0$, (Transversality condition)

(b) If $\vec{U}$ is of the form

$$
\vec{U}=\left\{\vec{u} \in\left(L^{2}(\Lambda, \mathbb{R})\right)^{3} \mid u_{i}(x) \in V_{i} \text {, a. e. on } \Lambda\right\} \text {, with } V_{i} \subset \mathbb{R}, i=1,2,3 \text {. }
$$

Then (28a) is equivalent to the minimum element wise (29), where:

$H_{\vec{v}}^{T} \cdot \vec{v}=\min _{\vec{u} \in \vec{V}} H_{\vec{v}}^{T} \cdot \vec{u}$ a.e. on $\Lambda$

Proof : (a) From theorem 4.2, the functional $Y_{\ell}(\vec{v})$ has a continuous FD at each $\vec{v} \in \vec{U}$, since the control $\vec{v} \in \vec{U}_{A}$ is optimal, then by K.T.L theorem there exist multipliers $\lambda_{\ell} \in \mathbb{R}$, $\ell=0,1,2$, with $\lambda_{0}, \lambda_{2} \geq 0, \sum_{\ell=0}^{2}\left|\lambda_{\ell}\right|=1$, such that $\left(\lambda_{0} \dot{\vec{Y}}_{0 \vec{v}}(\vec{v})+\lambda_{1} \dot{\vec{Y}}_{1 \vec{v}}(\vec{v})+\lambda_{2} \dot{\vec{Y}}_{2 \vec{v}}(\vec{v})\right) \cdot(\vec{u}-$ $\vec{v}) \geq 0, \forall \vec{u} \in \vec{U}$ and $\lambda_{2} Y_{2}(\vec{v})=0$, substituting the FD of $Y_{\ell}(\vec{v})(\forall \ell=0,1,2)$ in the above inequality, to get

$\int_{\Lambda}\left(\left(\zeta_{1}\left(a_{2 v_{1}}-a_{1 v_{1}}\right)+y_{1 v_{1}}\right) \delta v_{1}+\left(\zeta_{2}\left(p_{2 v_{2}}-p_{1 v_{2}}\right)+y_{2 v_{2}}\right) \delta v_{2}+\left(\zeta_{3}\left(k_{2 v_{3}}-k_{1 v_{3}}\right)+\right.\right.$ $\left.\left.y_{3 v_{3}}\right) \delta v_{3}\right) d x \geq 0$ 
where $\zeta_{i}=\sum_{\ell=0}^{2} \lambda_{\ell} \zeta_{i \ell}, y_{i v_{i}}=\sum_{\ell=0}^{2} \lambda_{\ell} y_{i \ell v_{i}}$, for $i=1,2,3$,

$\Rightarrow \int_{\Lambda} H_{\vec{v}}{ }^{T} \cdot \overrightarrow{\delta v} d x \geq 0, \forall \vec{u} \in \vec{U} \overrightarrow{\delta v}=\vec{u}-\vec{v}$

(b) Let $\vec{U}=\left\{u \in\left(L^{2}(\Lambda, \mathbb{R})\right)^{3} \mid u_{i}(x) \in V_{i}\right.$, a. e. on $\left.\Lambda\right\}$, with $V_{i} \subset \mathbb{R}, i=1,2,3, \mu$ is a

"Lebesgue" measure on $\Lambda,\left\{v_{n}\right\}$ be a sequence in $\vec{U}_{\vec{V}}$ and assume $S \subset \Lambda$ be a measurable set such that $\vec{u}(x)=\left\{\begin{array}{c}\vec{u}_{n}(x), \text { if } x \in S \\ \vec{v}(x), \text { if } x \notin S\end{array}\right.$. Hence (28a), becomes

$\int_{S} H_{\vec{v}}{ }^{T} \cdot\left(\vec{u}_{n}-\vec{v}\right) d x \geq 0$, for each such set $S \Longrightarrow{H_{\vec{v}}}^{T} .\left(\vec{u}_{n}-\vec{v}\right) \geq 0$, a.e. on $\Lambda$

That is it satisfies in $\varphi$ with $\varphi=\bigcap_{n} \varphi_{n}$, where $\varphi_{n}=\Lambda-\Lambda_{n}$, with $\mu\left(\Lambda_{n}\right)=0$, but $\varphi$ is independent of $n$, with $\mu(\Lambda / \varphi)=0$ and since $\left\{\vec{v}_{n}\right\}$ is dense in $\vec{U}_{\vec{V}}$, then

$$
H_{\vec{u}}{ }^{T} \cdot(\vec{u}-\vec{v}) \geq 0 \text {, a.e. on } \Lambda \Longrightarrow H_{\vec{v}}{ }^{T} \cdot \vec{v}=\min _{\vec{u} \in \vec{V}} H_{\vec{v}}{ }^{T} \cdot \vec{u} \text { a.e. on } \Lambda \text {. }
$$

\section{Theorem 5.3: Optimality Sufficient Conditions:}

In addition to the hypotheses $\mathrm{A}, \mathrm{B} \& \mathrm{C}$, with $\vec{U}$ is convex , $\left(a_{1} \& y_{11}\right),\left(p_{1} \& y_{12}\right),,\left(k_{1} \& y_{13}\right)$ are affine w.r.t $\left(\xi_{1}, v_{1}\right),\left(\xi_{2}, v_{2}\right),\left(\xi_{3}, v_{3}\right)$, resp $a_{2}, p_{2}, k_{2}$ are affine w.r.t $v_{1}, v_{2}, v_{3}$ resp for each $x$ , and $y_{\ell i},(\ell=0,2, i=1,2,3)$ is convex w.r.t. $\left(\xi_{i}, v_{i}\right)$ for each $x$. Then the necessary conditions in theorem5.2, with $\lambda_{0}>0$, are also sufficient.

Proof: suppose

$$
\begin{array}{ll}
a_{1}\left(x, \xi_{1}, v_{1}\right)=a_{11}(x) \xi_{1}+a_{12}(x) v_{1}+a_{13}(x), & a_{2}\left(x, v_{1}\right)=a_{21}(x) v_{1}+a_{22}(x), \\
p_{1}\left(x, \xi_{2}, v_{2}\right)=p_{11}(x) \xi_{2}+p_{12}(x) v_{2}+p_{13}(x), & p_{2}\left(x, v_{2}\right)=p_{21}(x) v_{2}+p_{22}(x), \\
k_{1}\left(x, \xi_{3}, v_{3}\right)=k_{11}(x) \xi_{3}+k_{12}(x) v_{3}+k_{13}(x), & k_{2}\left(x, v_{3}\right)=k_{21}(x) v_{3}+k_{22}(x),
\end{array}
$$

And that $\vec{v} \in \vec{U}_{A}, \vec{v}$ is satisfied the K.T.L. and the Transversality condition i.e.

$\int_{\Lambda} H_{\vec{v}}(x, \vec{\xi}, \vec{\zeta}, \vec{v}) \cdot \overrightarrow{\delta v} d x \geq 0, \forall \vec{u} \in \vec{U} \quad$ and $\quad \lambda_{2} y_{2}(\vec{v})=0$

Let $Y(\vec{v})=\sum_{\ell=0}^{2} \lambda_{\ell} y_{\ell}(\vec{v})$, then $\dot{\vec{Y}}(\vec{v}) \overrightarrow{\delta v}=\sum_{\ell=0}^{2} \lambda_{\ell} \dot{\vec{Y}}_{\ell}(\vec{v}) \overrightarrow{\delta v}$

$$
\begin{aligned}
= & \sum_{\ell=0}^{2} \lambda_{\ell} \int_{\Lambda}\left[\left(\zeta_{1 \ell}\left(a_{2 v_{1}}-a_{1 v_{1}}\right)+y_{1 \ell v_{1}}\right) \delta v_{1}+\left(\zeta_{2 \ell}\left(p_{2 v_{2}}-p_{1 v_{2}}\right)+y_{2 \ell v_{2}}\right) \delta v_{2}\right. \\
& \left.+\left(\zeta_{3 \ell}\left(k_{2 v_{3}}-k_{1 v_{3}}\right)+y_{3 \ell v_{3}}\right) \delta v_{3}\right) d x=\int_{\Lambda} H_{\vec{v}}(x, \vec{\xi}, \vec{\zeta}, \vec{v}) \cdot \overrightarrow{\delta v} d x \geq 0
\end{aligned}
$$

Let $\left(v_{1}, v_{2}, v_{3}\right)$ and $\left(\bar{v}_{1}, \bar{v}_{2}, \bar{v}_{3}\right)$ are two given controls, then $\left(\xi_{1}=\xi_{1 v_{1}}, \xi_{2}=\xi_{2 v_{2}}, \xi_{3}=\right.$ $\left.\xi_{3 v_{3}}\right)$ and $\left(\bar{\xi}_{1}=\bar{\xi}_{1 v_{1}}, \bar{\xi}_{2}=\bar{\xi}_{2 v_{2}}, \bar{\xi}_{3}=\bar{\xi}_{3 v_{3}}\right)$ are their corresponding solutions, substituting the pair $(\vec{v}, \vec{\xi})$ in (1)-(4) and multiplying the obtained equation by $\kappa \in[0,1]$ once and once again the pair $(\overrightarrow{\vec{v}}, \vec{\xi})$ in (1)-(4) multiplying the obtained equation by $(1-\kappa)$, finally then blending together the obtained equations from each corresponding equations once get:

$$
\begin{aligned}
& -B_{1}\left(\kappa \xi_{1}+(1-\kappa) \bar{\xi}_{1}\right)+\left(\kappa \xi_{1}+(1-\kappa) \bar{\xi}_{1}\right)-\left(\kappa \xi_{2}+(1-\kappa) \bar{\xi}_{2}\right)-\left(\kappa \xi_{3}+(1-\kappa) \bar{\xi}_{3}\right) \\
& +a_{11}(x)\left(\kappa \xi_{1}+(1-\kappa) \bar{\xi}_{1}\right)+a_{12}(x)\left(\kappa v_{1}+(1-\kappa) \bar{v}_{1}\right)+a_{13}(x)=a_{21}(x)\left(\kappa v_{1}+\right. \\
& \left.(1-\kappa) \bar{v}_{1}\right)+a_{22}(x) \\
& \kappa \xi_{1}+(1-\kappa) \bar{\xi}_{1}=0 \\
& -B_{2}\left(\kappa \xi_{2}+(1-\kappa) \bar{\xi}_{2}\right)+\left(\kappa \xi_{1}+(1-\kappa) \bar{\xi}_{1}\right)+\left(\kappa \xi_{2}+(1-\kappa) \bar{\xi}_{2}\right)+\left(\kappa \xi_{3}+(1-\kappa) \bar{\xi}_{3}\right)+ \\
& p_{11}(x)\left(\kappa \xi_{2}+(1-\kappa) \bar{\xi}_{2}\right)+p_{12}(x)\left(\kappa v_{2}+(1-\kappa) \bar{v}_{2}\right)+p_{13}(x)=p_{21}(x)\left(\kappa v_{2}+\right. \\
& \left.(1-\kappa) \bar{v}_{2}\right)+p_{22}(x) \\
& \kappa \xi_{2}+(1-\kappa) \bar{\xi}_{2}=0
\end{aligned}
$$




$$
\begin{aligned}
& -B_{3}\left(\kappa \xi_{3}+(1-\kappa) \bar{\xi}_{3}\right)+\left(\kappa \xi_{1}+(1-\kappa) \bar{\xi}_{1}\right)-\left(\kappa \xi_{2}+(1-\kappa) \bar{\xi}_{2}\right)+\left(\kappa \xi_{3}+(1-\kappa) \bar{\xi}_{3}\right)+ \\
& k_{11}(x)\left(\kappa \xi_{3}+(1-\kappa) \bar{\xi}_{3}\right)+k_{12}(x)\left(\kappa v_{3}+(1-\kappa) \bar{v}_{3}\right)+k_{13}(x)=k_{21}(x)\left(\kappa v_{3}+\right. \\
& \left.(1-\kappa) \bar{v}_{3}\right)+k_{22}(x) \\
& \kappa \xi_{3}+(1-\kappa) \bar{\xi}_{3}=0
\end{aligned}
$$

Now, if we have the control vector $\overrightarrow{\bar{v}}=\left(\overline{\bar{v}}_{1}, \overline{\bar{v}}_{2}, \overline{\bar{v}}_{3}\right)$, with $\overline{\bar{v}}_{1}=\kappa v_{1}+(1-\kappa) \bar{v}_{1}$, $\overline{\bar{v}}_{2}=\kappa v_{2}+(1-\kappa) \bar{v}_{2}, \overline{\bar{v}}_{3}=\kappa v_{3}+(1-\kappa) \bar{v}_{3}$. Then from $(30 \mathrm{a} \& \mathrm{~b}),(31 \mathrm{a} \& \mathrm{~b}),(32$ $\mathrm{a} \& \mathrm{~b})$, once get that

$\overline{\bar{\xi}}_{1}=\xi_{1 \bar{v}_{1}}=\xi_{1\left(\kappa v_{1}+(1-\kappa) \bar{v}_{1}\right)}=\kappa \xi_{1}+(1-\kappa) \bar{\xi}_{1}$,

$\overline{\bar{\xi}}_{2}=\xi_{2 \bar{v}_{2}}=\xi_{2\left(\kappa v_{2}+(1-\kappa) \bar{v}_{2}\right)}=\kappa \xi_{2}+(1-\kappa) \bar{\xi}_{2}$,

$\overline{\bar{\xi}}_{3}=\xi_{3 \bar{v}_{3}}=\xi_{3\left(\kappa v_{3}+(1-\kappa) \bar{v}_{3}\right)}=\kappa \xi_{3}+(1-\kappa) \bar{\xi}_{3}$

are their corresponding solutions, i.e. $\left(\overline{\bar{\xi}}_{1}, \overline{\bar{\xi}}_{2}, \overline{\bar{\xi}}_{3}\right)$ is satisfied (1-4). So, the operator $v_{i} \mapsto \xi_{i v_{i}}$ is convex- linear w.r.t $\left(\xi_{i}, v_{i}\right)(i=1,2,3)$, for each $x \in \Lambda$.

Now, since $y_{1 i}\left(x, \xi_{i}, v_{i}\right)$ is affine w.r.t. $\left(\xi_{i}, v_{i}\right)$, for each $x \in \Lambda$ and from the convex -linear property of operators $v_{i} \mapsto \xi_{i v_{i}}$, once gets that $Y_{1}(\vec{v})$ is convex-linear w.r.t $(\vec{\xi}, \vec{v}), \forall x \in \Lambda$.

The convexity of $Y_{\ell}(\vec{v}) \quad$ (for $\ell=0,2$ ) w.r.t. $(\vec{\xi}, \vec{v})$, for each $x \in \Lambda$ is obtained from the hypotheses at each of $y_{\ell i}$ is convex w.r.t. $\left(\xi_{i}, v_{i}\right) \forall x \in \Lambda,(\forall \ell=0,2, \& i=1,2,3)$. Hence $Y(\vec{v})$ is convex w.r.t $(\vec{\xi}, \vec{v})$ in the convex set $\vec{U}=\vec{U}_{\vec{V}}$ and it has a continuous FD satisfied $\dot{\vec{Y}}(\vec{v}) \overrightarrow{\delta v} \geq 0 \Rightarrow Y(\vec{v})$ has a minimum at $\vec{v} \Rightarrow Y(\vec{v}) \leq Y(\vec{u}), \forall \vec{u} \in \vec{U} \Rightarrow$

$\lambda_{0} Y_{0}(\vec{v})+\lambda_{1} Y_{1}(\vec{v})+\lambda_{2} Y_{2}(\vec{v}) \leq \lambda_{0} Y_{0}(\vec{u})+\lambda_{1} Y_{1}(\vec{u})+\lambda_{2} Y_{2}(\vec{u})$

Now, let $\vec{u}$ be an admissible control and since $\vec{v}$ is also admissible and satisfies the Transversality condition, then (33) $\operatorname{becomes}_{0}(\vec{v}) \leq Y_{0}(\vec{u}), \forall \vec{u} \in \vec{U}$ i.e. $\vec{v}$ is an optimal control for the problem.

\section{Conclusion}

The existence and uniqueness theorem for the solution (continuous state vector) of the TNLEBVP is stated and proved successfully using the Mint-Browder theorem when the TCCOCV is given. Also, the existence theorem of a TCCOCV governing by the TNLEBVP is proved. The existence and uniqueness solution of the TAEqs related with the TNLEBVP is studied. The derivation of the FD of the Hamiltonian is obtained. Finally, the theorem of necessary conditions so as the sufficient condition theorem for optimality of the constrained problem are stated and proved.

\section{Acknowledgement}

I wish to express my sincere thanks to the University of Mustansiriyah for providing a pleasurable and stimulating environment to carry out research. Also, I would like to extend our sincere esteems to the College of Science- Department of Mathematics for their timely support.

\section{References}

1. Miele, A.; Weeks, M.W.; Ciarcia, M. Optimal Trajectories for Spacecraft Rendezvous, $J$ Optim Theory Appl.2007, 132, 353- 37.

2. Stodola, P.; Mazal, J. Optimal Location and Motion of Autonomous Unmanned Ground Vehicles. Wseas Transactions on Signal Processing.2010, 6, 2, 68-77. 
3. Lenhart, S.; Workman, J. Optimal Control Applied to Biological Models. Chapman \& Hall, CRC Press, USA, 2007.

4. Biswas, M.H.A.; Ara, M.; Haque, M.N.; Rahman, M.A. Application of Control Theory in the Efficient and Sustainable Forest Management. International Journal of Scientific and Engineering Research.2011, 3, 2.

5. Orpel, A. Optimal Control Problems with Higher Order Constraints. Folia Mathematica.2009, 16, 1, 31-44.

6. Al-Hawasy, J. The Continuous Classical Optimal Control of a Nonlinear Hyperbolic Equation (CCOCP). Al-Mustansiriyah Journal of Science.2008, 19, 8, 96-110, doi.org/10.23851/mjs.v29i1.159.

7. Chryssoverghi, I.; Al-Hawasy, J. The Continuous Classical Optimal Control Problem of a Semilinear parabolic Equations (CCOCP), Journal of Kerbala University.2010, 3,

8. Bors, D.; Walczak, S. Optimal control elliptic system with distributed and boundary controls. Nonlinear Analysis.2005, 63, e1367-e1376.

9. Al-Hawasy, J. The Continuous Classical Optimal Control of a Couple Nonlinear Hyperbolic Partial Differential Equations With Equality and Inequality Constraints. Iraqi Journal of Science.2016, 57, 2C, 1528-1538.

10. Al-Hawasy, J. The Continuous Classical Boundary Optimal Control of a Couple Nonlinear Hyperbolic Boundary Value Problem With Equality and Inequality Constraints, Accepted in Baghdad Science Journal, in, 2019.

11. Kadhem, G.M. The Continuous Classical Optimal Control Problem of Partial Differential Equations, M.Sc. Thesis, Al-Mustansiriyah University, Baghdad, Iraq, 2015.

12. Al-Hawasy, J.; Naeif A.H. The Continuous Classical Boundary Optimal Control of a Couple Linear of Parabolic Partial Differential Equations Al-Mustansiriyah, Journal of Science.2018, 29, 1, 118-126, doi.org/10.23851/mjs.v29i1.159.

13. Al-Rawdanee, E. H. M. The Continuous Classical Optimal Control Problem of a NonLinear Partial Differential Equations of Elliptic Type. M.Sc. Thesis, Al-Mustansiriyah University, Baghdad,Iraq, 2015.

14. Al-Hawasy, J.; Al-Qaisi S.J. The Continuous Classical Optimal Boundary Control of a Couple Linear Elliptic Partial Differential Equations, 1st Scientific International Conference, College of Science, Al-Nahrain University.2017, 21-22/11/2017, Part I, 137142, DOI. 10.22401/SIC.I.18.

15. Al-Hawasy, J.; Jasim D.k.J. The Continuous Classical Optimal Control Problems of a Triple Elliptic Partial Differential Equations, Submitted in Ibn Al-Haitham for pure and Applied Science, in.2019, doaj.org/toc/2521-3407.

16. Al-Hawasy, J.; Jaber M.A. The Continuous Classical Optimal Control governing by Triple Parabolic Boundary Value Problem, Submitted in Ibn Al-Haitham for pure and Applied Science, in.2019, doaj.org/toc/2521-3407.

17. Borzabadi, A.H.; Kamyad, A.V.; Farahi, M.H.G. Optimal Control of the Heat Equation in an Inhomogeneous Body, J. Appl. Math. and Computing. 2004, 15, 1$2,127-146$.

18. Chyssoverghi, I.; Bacopoulos, A. Approximation of Relaxed Nonlinear Parabolic Optimal Control Problems, Journal of Optimization Theory and Applications.1993, 77, 31-50. 
Ibn Al-Haitham Jour. for Pure \& Appl. Sci. 33 (3) 2020

19. Bacopoulos, A.; Chryssoverghi, I., "Numerical Solutions of Partial Differential Equations by Finite Elements Methods", Symeom Publishing Co, Athens, 1986.

20. Brezis, H. Functional Analysis, Sobolev Spaces and Partial Differential Equations: Springer, New York, USA, 2011. 